\title{
Soudan 2 Atmospheric Neutrino Oscillation Studies
}

\author{
Hugh Gallagher* for the Soudan2 Collaboration \\ Department of Physics and Astronomy \\ University of Minnesota \\ 116 Church St. SE \\ Minneapolis, MN 55455 USA \\ E-mail: 'gallag@hep.umn.edu'
}

Abstract: A 5.1 fiducial-kiloton year exposure of the Soudan 2 detector has been analyzed for evidence of neutrino oscillations. The flavor-ratio is $R=\frac{\left(\nu_{\mu} / \nu_{e}\right)_{\text {data }}}{\left(\nu_{\mu} / \nu_{e}\right)_{M C}}=$ $0.68 \pm 0.11$ (stat) \pm 0.06 (syst.). A number of data sets, with different sensitivities to neutrino oscillations, are presented and their use in the analysis described. Under the hypothesis of $\nu_{\mu} \rightarrow \nu_{\tau}$ oscillations the data likelihoods are determined and fits to oscillation parameters are carried out using the Feldman-Cousins method.

\section{The Detector and Data Reduction}

The Soudan 2 detector is a 963 ton fine-grained tracking calorimeter located at a depth of 2100 meters-water-equivalent in the Soudan Mine State Park in Soudan, Minnesota. The data analyzed for this report comprise 5.1 fiducial-kiloton years of the total detector exposure. The detector itself is a modular, slow drift calorimeter. The drift elements are $1 \mathrm{~m}$ long hytrel tubes, embedded in a stack of thin corrugated steel plates which form a honeycomb-like structure. The entire detector is made of $2241 \mathrm{~m} \mathrm{x} 1 \mathrm{~m} \times 2.7 \mathrm{~m}$ modules which weigh 4.3 tons, and is surrounded by a nearly hermetic veto shield of proportional tubes.

Events are classified in Soudan 2 on the basis of their topology, which is determined through a physicist scan. Events are placed in one of three categories: tracks, showers, or multiprongs, which are meant to encompass quasi- elastic $\nu_{\mu}$, quasi-elastic $\nu_{e}$, and inelastic events, respectively. All tracks and showers in each event are identified by hand using a graphical display program which interfaces to the experiment reconstruction code. The flavor assignment for multiprong events is based on a leading prong algorithm: an event with a leading, non-scattering track with ionization consistent with a muon is a candidate $\nu_{\mu}$ charged current (CC) event, an event having a prompt energetic shower is a candidate $\nu_{e} \mathrm{CC}$ event.

\footnotetext{
${ }^{*}$ Speaker.
} 


\begin{tabular}{|l|cc|}
\hline & Tracks & Showers \\
\hline Data & 133 & 193 \\
Monte Carlo & 173.1 & 160.3 \\
Bkgd Corrected Data & $105.1 \pm 12.7$ & $142.3 \pm 13.9$ \\
\hline
\end{tabular}

Table 1: Track and Shower Samples from a 5.1 fiducial kiloton-year exposure.

\section{The Flavor Ratio}

We determine the double ratio $R=\frac{\left(\nu_{\mu} / \nu_{e}\right)_{d a t a}}{\left(\nu_{\mu} / \nu_{e}\right)_{M C}}$ after carrying out a correction to remove background from each sample. The background comes from neutrons and photons which enter the detector and interact unaccompanied by any activity in the veto shield. Events caused by cosmic-ray induced backgrounds generally produce hits in the veto shield; these 'tagged background' events are used to characterize the background. The amount of background is determined by fitting event vertex depth distributions to a sum of neutrino Monte Carlo (MC) and tagged background events. The general method is described in more detail in Ref [i] $\left[\begin{array}{l}1 \\ 1\end{array}\right]$. The track and shower samples before and after background corrections are given in Table $i_{-i}^{1}$ The flavor ratio is then:

$$
R=\frac{\left(\nu_{\mu} / \nu_{e}\right)_{d a t a}}{\left(\nu_{\mu} / \nu_{e}\right)_{M C}}=0.68 \pm 0.11(\text { stat. }) \pm 0.06(\text { syst. })
$$

\section{Data Sets}

Neutrino oscillations affect atmospheric neutrino measurements in several ways; they result in an anomalous flavor ratio and can also affect the $\mathrm{L} / \mathrm{E}, \mathrm{E}$, or zenith angle distributions of the events, where $\mathrm{L}$ is the distance the neutrino has travelled and $\mathrm{E}$ is the neutrino energy. The analysis carried out here divides events into two categories, high-resolution and low-resolution, based on the reliability of the $\mathrm{L} / \mathrm{E}$ measurement. In

\begin{tabular}{|l|rr|}
\hline & $\nu_{\mu}$ CC & $\nu_{e}$ CC \\
\hline High-Res: & & \\
Data & 107.0 & 134.0 \\
Bkgd Corrected Data & 99.5 & 128.7 \\
MC & 169.0 & 155.7 \\
\hline Low-Res: & & \\
Data & 79.0 & 122.0 \\
Bkgd Corrected Data & 48.2 & 74.8 \\
MC & 63.8 & 70.6 \\
\hline
\end{tabular}

Table 2: Breakdown of $\nu_{\mu} \mathrm{CC}$ and $\nu_{e} \mathrm{CC}$ events into low and high resolution categories. this approach, the high-resolution events are used for the L/E fits, while low-resolution events are included in the fits only through their contribution to the flavor ratio. MC studies show that the flavor assignment for low- resolution events is generally correct: $93.6 \%$ of low-resolution $\nu_{\mu} \mathrm{CC}$ events are called tracks and $95.5 \%$ of low-resolution $\nu_{e} \mathrm{CC}$ events are called showers. For the analysis described here, only low-resolution tracks and showers are used.

The high-resolution data set includes events from both the quasi-elastic (track and shower) and multiprong topologies. Quasi-elastic events pass the high-resolution cuts if they have a recoil proton and $p_{\text {lept }}>150 \mathrm{MeV} / \mathrm{c}$, or, if a recoil is not visible, the single visible lepton must have $E_{v i s}>600 \mathrm{MeV}$. In order to pass the high-resolution cuts, mul- 

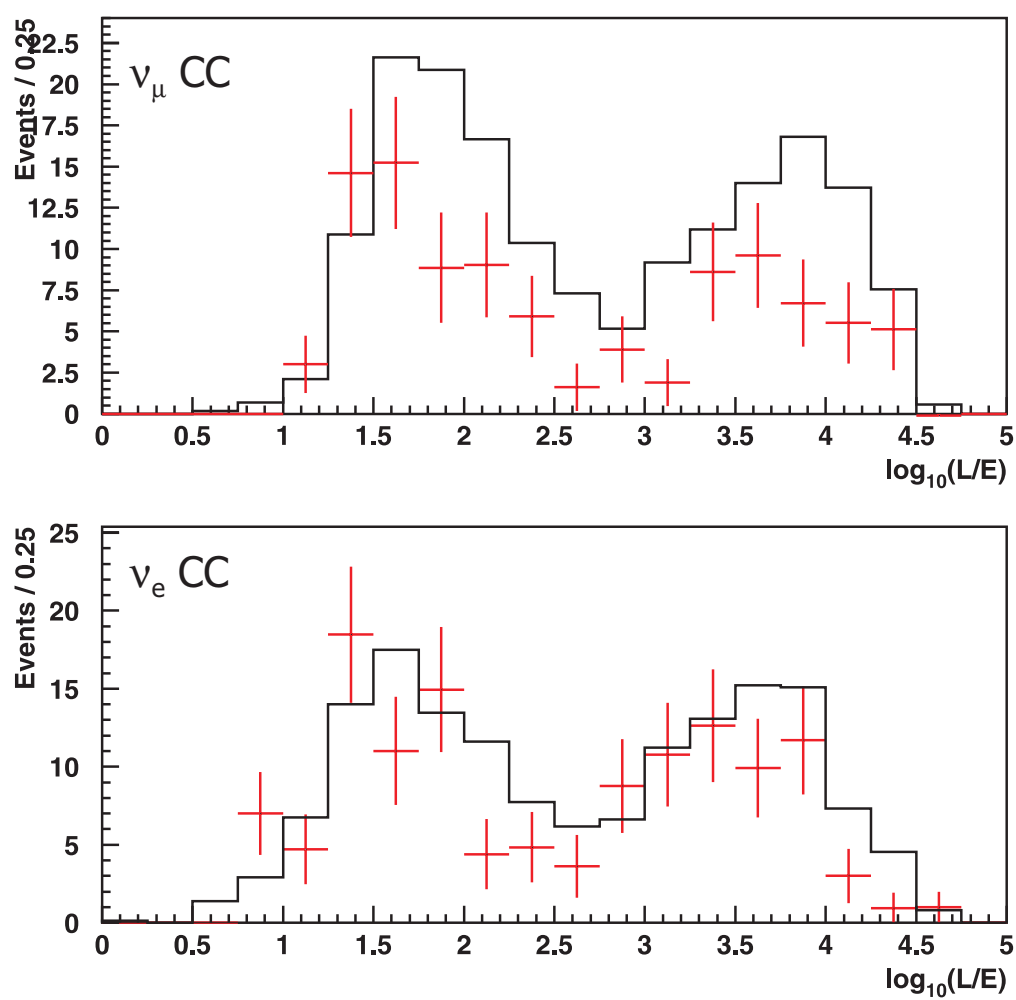

Figure 1: L/E distributions for high-resolution events. The crosses are the data, solid histogram is the Monte Carlo + the expected amount of background as determined from the depth fits. The Monte Carlo is normalized to the prediction of Bartol 96 [2].

tiprong events must have $E_{v i s}>700 \mathrm{MeV} / \mathrm{c}$, have a total visible 4 -momentum $p_{\text {vis }}>450$ $\mathrm{MeV} / \mathrm{c}$, and a final state lepton momentum $p_{\text {lep }}>250 \mathrm{MeV} / \mathrm{c}$. The resolution in $\log \frac{L}{E_{\nu}}$ is better than 0.5 for the high-resolution sample. Table $\underset{2}{2}$ gives the breakdown of events into high and low resolution categories. Background amounts in the $\nu_{\mu} \mathrm{CC}$ and $\nu_{e} \mathrm{CC}$ categories are determined using the depth fitting technique described previously. The higher energy data of the high-resolution set have little background: $7.0 \%(4.0 \%)$ of the $\nu_{\mu} \mathrm{CC}$ $\left(\nu_{e} \mathrm{CC}\right)$ samples as opposed to $39.0 \%(38.7 \%)$ of the lo-resolution $\nu_{\mu} \mathrm{CC}\left(\nu_{e} \mathrm{CC}\right)$ samples.

The Monte Carlo sample represents $\sim 31$ kiloton years of exposure. The flux model employed is that of Reference [i2]]. The MC sample used here differs from that employed in previous analyses in two ways. The normalization of the MC sample differs due to a correction in the bookkeeping procedures related to event generation. The normalization of the MC sample to the detector exposure used here is $8 \%$ lower than that used previously. A second correction is related to the simulation of electromagnetic showers in the Soudan 2 calorimeter. The tracking simulations that are part of the Soudan 2 Monte Carlo are based on EGS3 and only track particles to $1 \mathrm{MeV}$ [3i]. This threshold is considerably higher than the energy deposition required to produce a hit in a drift tube. Studies have shown that the net effect of the high tracking threshold on the simulation of electromagnetic showers is that the Monte Carlo produces $16 \%$ too few hits, independent of shower energy. We corrected this problem by scaling the numbers of hits in MC showers up by $16 \%$ 葡. The 
event energies are then re-calculated using the new numbers of shower hits. As a result of this correction the data and MC shower hit and energy distributions are now in good agreement. Neither of the corrections described here produce any significant changes in the results of the oscillation fits, as compared to previous analyses when performed on the same data set.

\section{Neutrino Oscillation Analysis}

The background corrected neutrino data are analyzed in terms of a $\nu_{\mu} \rightarrow \nu_{\tau}$ neutrino oscillation hypothesis. Figure $\underline{1}_{i}^{1}$ shows the L/E distributions for $\nu_{\mu} \mathrm{CC}$ and $\nu_{e}$ hi-resolution data events compared with the predictions of the Monte Carlo. In order to determine the region of $\left(\Delta m^{2}, \sin ^{2}(2 \theta)\right)$ parameter space consistent with the data, we perform a FeldmanCousins fit based on the data likelihood:

$$
\log P(\text { data })=\sum_{i=1}^{22} \log \frac{e^{-\mu_{i}} \mu_{i}^{N_{i}}}{N_{i} !}-\frac{1}{2}\left(\frac{f-f_{\text {Bartol }}}{0.20 f_{\text {Bartol }}}\right)^{2}
$$

The summation is over 20 bins in $\nu_{\mu}$ hi-resolution CC L/E, one bin for $\nu_{\mu}$ low-resolution $\mathrm{CC}$ events, and one bin for all $\nu_{e} \mathrm{CC}$ events (high+low resolution). $\mu_{i}\left(N_{i}\right)$ are the expected (measured) number of events in bin i. The final term comes from our knowledge of the MC normalization, which is included in the fits with an error of $20 \%$.

Five hundred Monte Carlo experiments are carried out at each point on a $40 \mathrm{x} 40$ grid of $\left(\Delta m^{2}, \sin ^{2}(2 \theta)\right)$ space. The range of parameter space covered is visible in Figure 2.2 For each MC experiment, a simulated data set is created which models the data sample one could obtain if neutrino oscillations occur with the oscillation parameters of that point. In each $\mathrm{MC}$ experiment, data and background samples are subjected to statistical fluctuations, and the fits are carried out exactly as performed on the real data. This allows one to calculate a likelihood value $L_{90}$ for each point of $\left(\Delta m^{2}, \sin ^{2}(2 \theta)\right)$ parameter space, which is the value of the likelihood below which one would expect $90 \%$ of the experiments to fall if the true value of the oscillation parameters are the values at that point [is. This method also allows for the inclusion of some systematic errors through the use of 'nuisance parameters'. These parameters correspond to parameters in the analysis which contain some errors. For each $\mathrm{MC}$ experiment, the value for the parameter is selected from a gaussian distribution. In this way this analysis incorporates the systematic errors due to the error on the electromagnetic energy scaling and the background subtraction.

The best fit to the data occurs at $\left(\Delta m^{2}, \sin ^{2}(2 \theta)\right)=\left(8.9 \times 10^{-3} e V^{2}, 0.94\right)$. For the best fit point the value of the MC normalization is 0.160 , which is 0.96 of the MC normalization given by the Bartol 96 fluxes [2] value of $L_{90}$ determines whether a point is inside or outside the confidence interval. The $90 \%$ confidence interval thus obtained is shown in Figure 2

\section{Future Plans}

In addition to the data set described here, we have isolated an additional sample of 31 partially contained events with $\nu_{\mu}$ flavor that will be included in oscillation analysis in the 


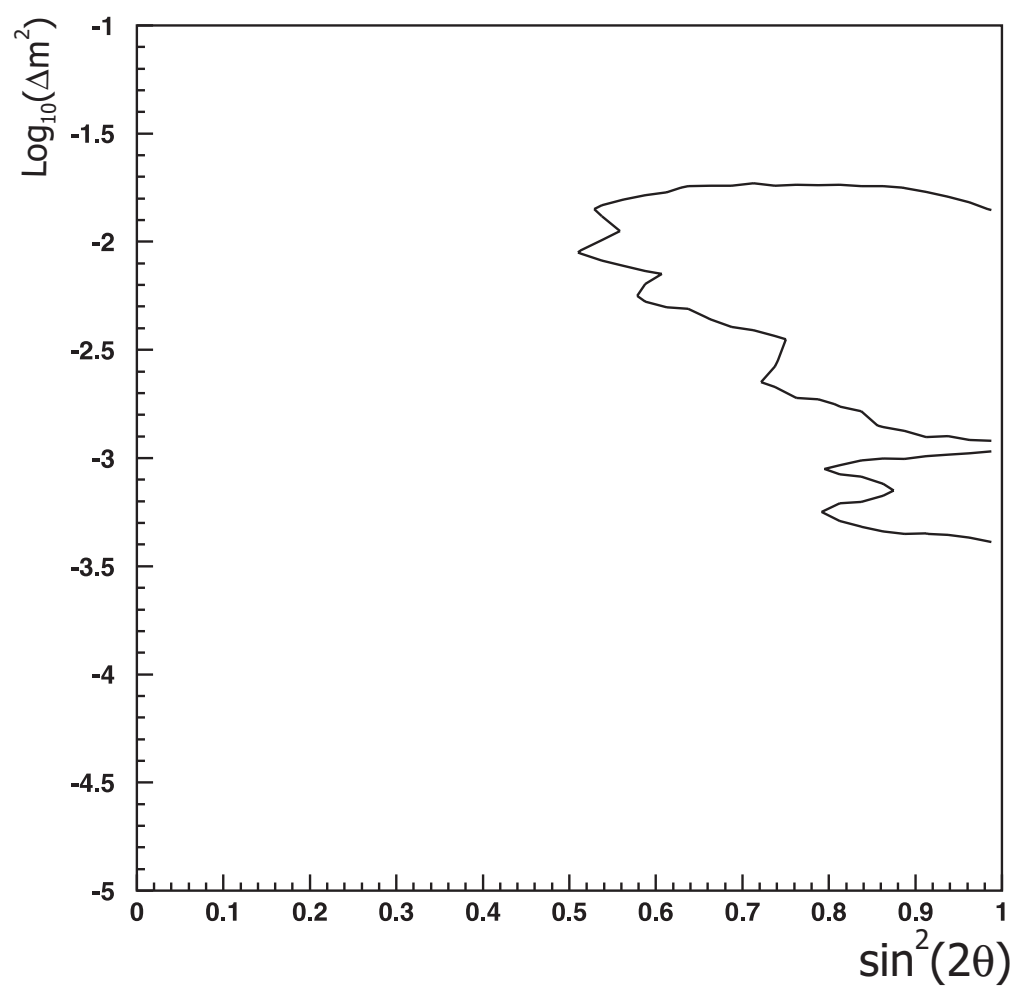

Figure 2: The $90 \%$ confidence level region of parameter space for $\nu_{\mu} \rightarrow \nu_{\tau}$ oscillations as determined from a Feldman-Cousins fit to Soudan 2 atmospheric neutrino contained event data.

near future. Low-resolution multiprongs will also be available for use in future analyses. Work is also underway to isolate upward going stopping muons, which are also sensitive to neutrino oscillations.

Data-taking for the Soudan 2 experiment ceased at end June 2001. The detector was turned off and placed into an inactive 'mothball' state where it has remained - completely intact - since that time. By proceeding in this way, the MINOS experiment retains the option to turn the tracking calorimeter back on when the NuMI neutrino beam becomes operative, should the experimental situation warrant the detector's revival.

\section{References}

[1] W.W.M. Allison et al., Phys. Lett. B 391(1997) 491; Phys. Lett. B 449 (1999) 137.

[2] V. Agrawal, T.K. Gaisser, P. Lipari, and T. Stanev, Phys. Rev. D 53 (1996) 1313.

[3] Walter R. Nelson, Hideo Hirayama, and David W.O. Rogers. The EGS4 Code System. SLAC, Stanford, California, December 1985.

[4] B. Speakman and H. Gallagher, Soudan 2 Internal Memos PDK-769/774, 2001.

[5] G.J. Feldman and R.D. Cousins, Phys. Rev. D 57 (1998) 3873. 